\title{
Braconnages identitaires sur la côte du Pacifique
}

\author{
Guy Poirier \\ Université de Waterloo
}

L'idée d'étudier les braconnages identitaires sur la côte du Pacifique et plus précisément en Colombie-Britannique m'est venue à la lecture de recueils de nouvelles dont je ne suis jamais parvenu à bien expliquer la composition. Dans un article publié récemment (Poirier, 2009), j'ai ainsi tenté de démontrer que les écrivains francophones dans cette province à l'extrême ouest de notre pays s'inspiraient de lieux de mémoire qui pouvaient être rattachés à deux principales catégories : les endroits connus ou visités dans le passé, alors qu'ils n'avaient pas encore quitté leur terre natale, et les espaces liés à leur province d'adoption. Contrairement au mouvement géographique qui coïncidait avec 
leur immigration ou leur changement de domicile, le déplacement des lieux d'intrigue s'était effectué par étapes. De façon concrète, on peut ainsi compter, dans la plupart des recueils de nouvelles publiés dans les années 1980 et 1990, un plus grand nombre d'histoires se déroulant dans l'espace correspondant à leur pays ou leur province d'origine. Ce phénomène se résorbe cependant dans les années 2000, et l'on assiste à l'émergence d'un nouveau rapport identitaire entre le soi et l'autre, que je rapproche de la notion de braconnage identitaire définie par Simon Harel : «Braconner, c'est savoir que l'autre a un territoire et que nous n'en avons pas » (p. 55). Pourquoi ce rapprochement, alors que Harel fait d'abord référence, dans sa théorie, à l'histoire littéraire du Québec? C'est qu'à mon avis, les écrivains francophones de la ColombieBritannique tentent présentement de prendre possession symboliquement - d'une partie du territoire de leur province d'accueil. S'insinuer ainsi dans la mosaïque culturelle implique donc l'utilisation de ruses qui permettront de faire coïncider l'expérience de migrant avec une réalité nouvelle. Le processus ne pouvant s'effectuer que très lentement, le recueil de nouvelles avec ses multiples facettes laisse entrevoir une progression oblique dans ce champ miné du rapport au territoire identitaire.

Dans cet article, qui porte plus directement sur le rapport identitaire entre le soi et l'autre et les dynamiques des braconnages identitaires, j'ai décidé d'explorer de façon plus précise la composition d'une série de sept recueils de nouvelles publiés entre 1988 et 2007 par des auteurs qui établirent domicile à différentes dates sur la côte ouest, mais qui gardent de très vifs souvenirs de leur vie antérieure en Europe, en Afrique ou dans les Prairies canadiennes. Mon corpus se 
compose du Totem(1988) de Marguerite-A. Primeau, des Nouvelles d'ici... et d'ailleurs (1995) de Christian Recizac, de Ol'Man, Ol'Dog et l'enfant ou autres nouvelles (1996) de Marguerite-A. Primeau, du Tableau rouge (1997) d'Inge Israël, De parts et d'autres de Claude Bouygues (1999) et de deux recueils de Jean-Claude Castex, Le Fantôme et autres histoires vraies (2005) et C'est arrivé un jour! (2007). Ce qui m'étonne, chez ces quatre auteurs, c'est que le rapport au passé et au présent s'inscrit dans leur œuvre un peu de la même manière, et ce, à plusieurs égards. C'est comme si le passé, personnel, dense, parfois tragique, impliquait nécessairement une réflexion humaniste, alors que le présent s'avérait superficiel et même mécanique dans sa construction des personnages et l'architecture des intrigues. De plus, une sémiotique de l'utilisation du retournement et de l'utilisation des objets inanimés se remarque dans les nouvelles dont l'action se déroule à notre époque.

De façon à explorer l'imbrication de ces univers, celui du soi et celui de l'autre, celui du passé et celui du présent, celui de l'ailleurs et celui de la Colombie-Britannique, j'ai d'abord décidé d'analyser l'architecture des sept recueils de mon corpus et de classifier les nouvelles qui y furent regroupées. Dans un deuxième temps, je vais faire ressortir la dimension personnelle et humaniste des nouvelles du passé, celles d'avant l'immigration au Canada. Finalement, je vais démontrer de quelle façon les nouvelles se déroulant en ColombieBritannique mettent en forme un univers différent des premières, et la manière dont les auteurs y tronquent leur plume d'écrivain pour celle du braconnier en quête d'un objet de récit. 


\section{Les recueils de nouvelles}

Les sept recueils choisis pour mon étude forment un corpus que je crois assez homogène. Les auteurs, femmes et hommes, ont vécu la première partie de leur existence dans une province ou un pays autre que la Colombie-Britannique, et ont choisi de joindre en un même ouvrage des nouvelles abordant ces deux univers. Ainsi, Marguerite-A. Primeau est née en Alberta où elle a enseigné de nombreuses années avant de s'installer en Colombie-Britannique. Christian Recizac se dit «citoyen du monde ${ }^{1}$ », et est également venu s'établir en ColombieBritannique après un long séjour en Afrique noire anglophone. Inge Israël, née en Allemagne, a grandi en France et en Irlande, et a vécu au Danemark avant de venir au Canada. Claude Bouygues, né dans le Tarn, a d'abord fait carrière en Tunisie et aux États-Unis avant de venir s'établir à Vancouver. Et, finalement, Jean-Claude Castex est originaire d'Algérie et habite l'Ouest canadien depuis 1976. Il faut bien comprendre que ces recueils, dans la typologie des œuvres de la ColombieBritannique, annoncent une littérature en devenir. Kathleen Kellett-Betsos aborde d'ailleurs cette question dans un article récent en traitant de ces livres sous une même rubrique intitulée « Des nouvellistes franco-colombiens en vedette ». Elle rappelle ainsi que

la nouvelle franco-colombienne s'inscrit à l'intérieur d'une institution littéraire émergente qui donne une voix à une communauté francophone produite de diasporas diverses, mais reliée aux autres communautés franco-canadiennes par un souci commun de soutenir la langue française et les cultures francophones. (p. 227)

\footnotetext{
1 Voir la quatrième de couverture de son recueil Nouvelles d'ici... et d'ailleurs.
} 
Précisons finalement que j'ai écarté de mon corpus des recueils très importants, mais qui représentent, selon moi, un autre type d'œuvres de création. Dans un premier temps, j'ai mis de côté le recueil publié sous la direction de Marie-France Hautberg intitulé Anthologie de nouvelles francophones de la côte-Pacifique du Canada. Il s'agit en fait d'un regroupement de nouvelles écrites par différents auteurs, et même si les récits se déroulent en Colombie-Britannique, des plumes différentes s'entremêlent et s'éloignent d'une architecture de recueil propre à un seul écrivain. J'ai aussi écarté les recueils de JeanClaude Boyer publiés sous le titre Nouvelles d'ici et d'ailleurs, car ces nouvelles se rapprochent plutôt des carnets de voyage. Ont finalement aussi été mis de côté quelques recueils de littérature jeunesse, comme le Gros lot de Jean-Claude Castex.

Afin d'illustrer l'architecture et la composition d'ensemble des recueils étudiés, je présente plus bas un tableau récapitulatif selon des thématiques qui serviront de base à mes analyses des braconnages littéraires. En effet, les nouvelles dont l'action se situe à l'extérieur du territoire de la ColombieBritannique vont constituer pour le propre de cette étude la littérature du soi, car il s'agit de points de référence mémoriels pour les écrivains. En revanche, les récits qui se déroulent en Colombie-Britannique seront considérés comme de la littérature de braconnage. Deux autres catégories apparaissent dans mon tableau: les nouvelles dont l'action se déroule au présent ou dans un passé récent et ailleurs qu'en ColombieBritannique, et les nouvelles que l'on ne peut classer ou dont le lieu n'est pas vraiment précisé. Ces deux catégories, si elles permettent d'identifier des particularités (il est évident que le recueil de Christian Recizac se rapproche du carnet d'anecdotes de voyage), ne s'insèrent pas, du moins pour l'instant, dans la 
perspective théorique du présent article. Notons finalement la lente apparition des nouvelles se déroulant en ColombieBritannique ou abordant un aspect important lié à la vie dans cette province. Alors que l'on ne peut en isoler qu'une, dans le recueil de Marguerite-A. Primeau, en 1988, on en compte six dans le recueil de 2007 de Jean-Claude Castex. Le braconnage littéraire s'amplifie donc, avec le temps, tout en maintenant des constantes, comme on le verra dans la troisième section de ma démonstration.

\section{Tableau (les nouvelles sont numérotées selon l'ordre} d'apparition dans le recueil)

\begin{tabular}{|c|c|c|c|c|}
\hline Recueil & $\begin{array}{l}\text { Nouvelles dont } \\
\text { l'action se dé- } \\
\text { roule complète- } \\
\text { ment ou en par- } \\
\text { tie au passé et } \\
\text { ailleurs qu'en } \\
\text { Colombie-Bri- } \\
\text { tannique (litté- } \\
\text { rature du soi) }\end{array}$ & $\begin{array}{l}\text { Nouvelles dont } \\
\text { l'action se } \\
\text { déroule au } \\
\text { présent et en } \\
\text { Colombie- } \\
\text { Britannique } \\
\text { (littérature de } \\
\text { braconnage) }\end{array}$ & $\begin{array}{l}\text { Nouvelles } \\
\text { dont l'action } \\
\text { se déroule au } \\
\text { présent ou } \\
\text { dans un passé } \\
\text { récent et } \\
\text { ailleurs qu'en } \\
\text { Colombie- } \\
\text { Britannique }\end{array}$ & $\begin{array}{l}\text { Nouvelles } \\
\text { que l'on ne } \\
\text { peut clas- } \\
\text { ser; nou- } \\
\text { velles dont } \\
\text { le lieu } \\
\text { n'est pas } \\
\text { vraiment } \\
\text { précisé }\end{array}$ \\
\hline $\begin{array}{l}\text { Marguerite- } \\
\text { A. Primeau, } \\
\text { Le Totem, } \\
1988\end{array}$ & $\begin{array}{l}\text { 2. «Paul } \\
\text { Polonais » } \\
\text { 3. « Les mille } \\
\text { "Ave Maria" de } \\
\text { ma grand-mère» } \\
\text { 4. « La maison } \\
\text { d'autrefois » }\end{array}$ & 1. « Le totem » & $\begin{array}{l}\text { 6. «Voici mes } \\
\text { mains, } \\
\text { Seigneur! » }\end{array}$ & $\begin{array}{l}\text { 5. « La } \\
\text { folle du } \\
\text { quartier» }\end{array}$ \\
\hline $\begin{array}{l}\text { Christian } \\
\text { Recizac, } \\
\text { Nouvelles } \\
\text { d'ici... et } \\
\text { d'ailleurs, } \\
1995\end{array}$ & $\begin{array}{l}\text { 15. « Effet } \\
\text { placebo » } \\
\text { 16. « Tout ou } \\
\text { rien » } \\
\text { 17. « C'est } \\
\text { mon tour » } \\
\text { 18. « Le vent } \\
\text { des cimes» }\end{array}$ & $\begin{array}{l}\text { 1. « Les bois de } \\
\text { mon cœur» } \\
\text { 3. « Le retour de } \\
\text { Poussy » }\end{array}$ & $\begin{array}{l}\text { 2. « Lino» } \\
\text { 4. «Un acci- } \\
\text { dent de trop » } \\
\text { 5. «Victor» } \\
\text { 6. « Pépé, Pé- } \\
\text { pito, pepitas » } \\
\text { 7. «P'tit } \\
\text { bonheur » } \\
\text { 8. «Un hom- } \\
\text { me heureux» } \\
\text { 9. «Simbad le } \\
\text { lion» }\end{array}$ & \\
\hline
\end{tabular}




\begin{tabular}{|c|c|c|c|c|}
\hline & & & $\begin{array}{l}\text { 10. «Automatic } \\
\text { express } \\
\text { service » } \\
\text { 11. «Ah! Les } \\
\text { belles photos » } \\
\text { 12. «Vive la } \\
\text { vi...deo » } \\
\text { 13. «Les piè- } \\
\text { ces détachées » } \\
\text { 14. «La peau } \\
\text { de l'ourse » } \\
\text { 19. «Les } \\
\text { fugitifs » } \\
\text { 20. «Jamais } \\
\text { deux sans toi » } \\
\text { 21. «L'Asiate» }\end{array}$ & \\
\hline $\begin{array}{l}\text { Marguerite- } \\
\text { A. Primeau, } \\
\text { Ol' Man, } \\
\text { Ol' Dog et } \\
\text { l'enfant et } \\
\text { autres } \\
\text { nouvelles, } \\
1996\end{array}$ & $\begin{array}{l}\text { 2. «Mon petit } \\
\text { ami“de" juif » } \\
\text { 3. «Granny » } \\
\text { 4. «Mémère } \\
\text { Desjarlais » } \\
\text { 5. « Les sapins } \\
\text { de Madame } \\
\text { Trotte-Menu » }\end{array}$ & $\begin{array}{l}\text { 1. « Ol' Man, } \\
\text { Ol’ Dog et } \\
\text { l'enfant » }\end{array}$ & & $\begin{array}{l}\text { 5. «Une } \\
\text { veille de } \\
\text { Noël» }\end{array}$ \\
\hline $\begin{array}{l}\text { Inge Israël, } \\
\text { Le tableau } \\
\text { rouge, } \\
1997\end{array}$ & $\begin{array}{l}\text { 3. «Tante Moll» } \\
\text { 4. « La main » } \\
\text { 5. « Sophia » } \\
\text { 6. « Le tableau } \\
\text { rouge » } \\
\text { 8. « Camilla » } \\
\text { 10. « La visite } \\
\text { du roi » } \\
\text { 11. « Lento » }\end{array}$ & $\begin{array}{l}\text { 1. « Les } \\
\text { ombres » } \\
\text { 12. « Le } \\
\text { nougat» }\end{array}$ & $\begin{array}{l}\text { 2. } \\
\text { "Échappée » } \\
\text { 7. « Pépita » } \\
\text { 9. « Le parc } \\
\text { de la paix » }\end{array}$ & \\
\hline $\begin{array}{l}\text { Claude } \\
\text { Bouygues, } \\
\text { De parts et } \\
\text { d'autres, } \\
1999\end{array}$ & $\begin{array}{l}\text { 1. « Le pre- } \\
\text { mier hameau » } \\
\text { 6. « Le mau- } \\
\text { vais rêve » } \\
\text { 7. « La visite } \\
\text { du grand } \\
\text { Schalitz vert. } \\
\text { Souvenirs de } \\
\text { l'Occupation » } \\
\text { 8. } \\
\text { «Trapabeluga }\end{array}$ & $\begin{array}{l}\text { 2. «Vancouver, } \\
\text { la ville du -ing » } \\
\text { 4. « Histoire } \\
\text { d'eau » } \\
\text { 5. « La } \\
\text { décadence. } \\
\text { Catalogue» }\end{array}$ & & $\begin{array}{l}\text { 3. «Le } \\
\text { pivert et } \\
\text { le } \\
\text { bouleau. } \\
\text { Fable- } \\
\text { récit» }\end{array}$ \\
\hline
\end{tabular}




\begin{tabular}{|c|c|c|c|}
\hline & $\begin{array}{l}\text { ou le vent des } \\
\text { histoires » }\end{array}$ & & \\
\hline $\begin{array}{l}\text { Jean-Claude } \\
\text { Castex, } \\
\text { Le fantôme } \\
\text { et autres } \\
\text { histoires } \\
\text { vraies, } \\
2005\end{array}$ & $\begin{array}{l}\text { 14. « Le } \\
\text { dernier } \\
\text { combat du } \\
\text { lieutenant » }\end{array}$ & $\begin{array}{l}\text { 1. « Le } \\
\text { fantôme » } \\
\text { 2. « Le congrès } \\
\text { de l'ACELF » } \\
\text { 3. « La femme } \\
\text { en or » } \\
\text { 4. «Amours } \\
\text { ancillaires » } \\
\text { 5. « Illusions } \\
\text { d'enfance » } \\
\text { 6. « Faux billets } \\
\text { doux» } \\
\text { 7. « Manuel le } \\
\text { macho » } \\
\text { 8. « Mélodie } \\
\text { d'amour » } \\
\text { 9. « Le pari » } \\
\text { 10. « Passion } \\
\text { libératrice » } \\
\text { 13. «Prises } \\
\text { d'otages » }\end{array}$ & $\begin{array}{l}11 . \\
\text { «Quelques } \\
\text { coups de } \\
\text { pédales de } \\
\text { trop...» } \\
\text { 12. « Le talon } \\
\text { d'Achille» }\end{array}$ \\
\hline $\begin{array}{l}\text { Jean-Claude } \\
\text { Castex, } \\
\text { C'est arrivé } \\
\text { un jour!, } \\
2007\end{array}$ & $\begin{array}{l}\text { 5. « La tache } \\
\text { d'encre » } \\
\text { 6. « L'homme } \\
\text { à la barbe } \\
\text { rousse » }\end{array}$ & $\begin{array}{l}\text { 1. « Christine» } \\
\text { 2. « Le petit } \\
\text { sous de cuivre » } \\
\text { 3. « L'eau c'est } \\
\text { la vie » } \\
\text { 4. « Jonas » } \\
\text { 7. } \\
\text { «L'évaluation» } \\
\text { 8. « La } \\
\text { contravention» }\end{array}$ & $\begin{array}{l}\text { 9. « Les } \\
\text { pèlerins » }\end{array}$ \\
\hline
\end{tabular}

Ce tableau répertoriant les lieux de l'intrigue des nouvelles permet d'affirmer que plusieurs recueils sont construits sur le même modèle d'ensemble. Ainsi, la ou les premières nouvelles, dans les cinq premiers recueils (ordre chronologique de parution) du corpus, se déroulent à Vancouver. Les nouvelles suivantes, dans trois cas sur sept, ont pour période temporelle le passé, alors que 
les nouvelles du recueil de Jean-Claude Recizac se situent ailleurs qu'en Colombie-Britannique. Dans un cas, la dernière nouvelle du recueil, question de boucler la boucle, se passe en ColombieBritannique. Finalement, les deux derniers recueils de la liste, publiés respectivement en 2005 et 2007 par Jean-Claude Castex, obéissent à des règles différentes. Dans les deux cas, la majorité des nouvelles se déroulent en Colombie-Britannique, le plus souvent au présent. Quelques nouvelles (trois, en tout), en fin et en milieu de recueil, se situent dans le passé du soi, alors qu'un nombre semblable se déroulent ailleurs qu'en ColombieBritannique.

Certaines similitudes entre les plans d'ensemble de ces recueils bicéphales peuvent donc être remarquées et annoncent un changement de facture dans les recueils qui vont paraître dans les prochaines années. Les publications lancées depuis le tournant du millénaire comportent ainsi un nombre de plus en plus grand de nouvelles se déroulant sur le territoire de la province, alors que ce type de récit se retrouve le plus souvent, dans les années qui précèdent, dans des positions stratégiques : au début, au milieu ou à la fin du recueil. Les recueils de nouvelles plus récents, du même coup, favorisent une narration d'événements contemporains; alors que les premières publications racontaient le soi, les plus jeunes s'attaquent donc à l'autre en le braconnant.

\section{Les nouvelles racontant le monde du soi}

J'entends, par les nouvelles racontant le soi, les récits se déroulant dans le passé et dans un lieu différent de la ColombieBritannique. L'impression qui s'en dégage rappelle la narration 
autobiographique. Pour Pierre Ouellet, par exemple, raconter le soi n'implique pas la narration de choses qui apparaissent dans l'immédiateté de notre vie, mais bien de celles qui

hantent notre mémoire et notre imagination autant que nos perceptions et nos affects pour trouver un sens que nous lui donnons ou prêtons bien plus que nous ne le découvrons et révélons. (p. 10)

En luttant contre "l'insignifiance foncière des choses" (ibid.), dans le présent, le sens du passé est source d'inspiration et de réflexion. La dimension autobiographique de ces nouvelles ne faisant pas de doute, plusieurs indices permettent d'en venir à une première typologie: nouvelle écrite au je, description de scènes en focalisation interne sur un personnage enfant, utilisation de personnages identifiés comme étant des membres de la famille ou de proches amis des narrateurs. Je compte ainsi quatre nouvelles, dans le recueil de Claude Bouygues, où l'on retrouve des termes comme oncle, grandmère, ou encore un personnage principal enfant qui raconte l'histoire. Dans l'œuvre d'Inge Israël, ce sont plutôt les lieux et les périodes temporelles qui renvoient à la vie d'un même groupe de personnes. Quatre nouvelles se déroulent ainsi en totalité dans le Paris d'avant ou d'après l'Occupation. Une nouvelle comprend, en abîme, un renvoi à Paris. Deux nouvelles se déroulent également en Irlande pendant la guerre. Les deux recueils de Jean-Claude Castex nous transportent à cinq reprises dans le passé, en France, laissant parfois croire à des souvenirs personnels («C'est mon tour») ou à des histoires liées à la famille (la grand-mère dans «Effet placebo »), et en Algérie («Le dernier combat du lieutenant»). Finalement, trois nouvelles de Marguerite-A. Primeau laissent entrevoir une filiation à des souvenirs d'enfance : «Paul polonais », « Les mille 
Ave Maria de ma grand-mère », " Mon petit ami de juif ». Bien entendu, il ne s'agit pas de prouver que ces nouvelles sont bel et bien des récits autobiographiques, mais plutôt de noter la présence de critères d'énonciation permettant de croire à une telle posture auctoriale.

Il faut également noter la profondeur de ces nouvelles qui sont les mieux réussies sur le plan du pathos, mais non pas nécessairement au niveau du retournement propre au genre que l'on pourrait y chercher. Par exemple, nombreuses sont les nouvelles qui se terminent de façon tragique : mort, humiliation ou disparition d'un jeune ami juif, sévices sur des enfants, pleurs. Il faut dire que ces nouvelles se déroulent en grande partie (et cela probablement à cause de l'âge des auteurs), avant, pendant, ou immédiatement après la Seconde Guerre mondiale. Les personnages évoluent également dans des milieux modestes, pauvres et même miséreux. Cela ne les empêche pas de maintenir un regard positif ou philosophe sur ces années. Rarement les personnages adultes suscitent la déception. Ce sont plutôt les jeunes, ceux qui ont quitté leur terre natale, qui éprouvent des remords. Dans la nouvelle éponyme «Le tableau rouge » d'Inge Israël, un personnage masculin retrouvé par la narratrice, quarante ans après la guerre, lui fait une proposition douteuse alors qu'il l'invite, à la fin du récit, à lui rendre visite à son atelier de Paris où il se rend souvent et où il est seul (Israël, 1997, p. 108).

Par ailleurs, toutes les nouvelles racontant le soi et qui font le plein de sens grâce au passé (réel ou imaginé) des narrateurs se greffent à des personnages phares qui donnent leur nom aux nouvelles. D'un côté, se trouvent les personnages « génériques ». Il s'agit du petit ami « de» juif (« Mon petit ami 
"de" juif », dans Primeau, 1996), de la grand-mère de l'un des personnages ( "Les mille "Ave Maria" de ma grand-mère », dans Primeau, 1988; "Effet placebo», dans Recizac, 1995), d'un prisonnier allemand, Hans, dont l'épouse juive ignore le passé hitlérien et qui apparaît dans la nouvelle «L'homme à la barbe rousse » de Jean-Claude Castex (notons cependant que cette nouvelle se déroule au Canada). Inge Israël va même plus loin sur cette pente en intitulant une nouvelle « La main ». Même si le lieu de cette nouvelle n'est pas indiqué clairement, il semble qu'elle se déroule dans une ville où les orphelins sont nombreux (probablement pendant la guerre) et vivent dans des conditions misérables dans un foyer mal surveillé par des dames d'une organisation charitable. L'homme tenant cet hospice, fort violent, est perçu par les enfants terrorisés comme étant « la main », et même la « main de Dieu ».

Il serait possible d'ajouter à ces personnages bien campés les narrateurs homodiégétiques que l'on retrouve dans les nouvelles à connotation autobiographique ou biographique; pensons à la nouvelle de Claude Bouygues, "Le mauvais rêve ", à celle de Jean-Claude Castex, «Le dernier combat du lieutenant », ou encore à celle de Christian Recizac, "C'est mon tour ». On retrouve en outre des personnages bien identifiés autour desquels se construit l'intrigue des nouvelles. Ces personnages donnent souvent leur nom au récit. Le lecteur sait donc, dès le départ, autour de qui s'organise la narration. Des personnages masculins et féminins partagent ce profil de vedette. Trapabeluga dans la nouvelle « Trapabeluga ou le vent des histoires » (Bouygues, 1999), Paul dans «Paul Polonais» (Primeau, 1988), Schalitz dans «La visite du grand Schalitz vert» (Bouygues, 1999). Ces personnages sont par ailleurs tous associés à une altérité: Trapabeluga est un véritable 
personnage de conte fantastique; Paul est juif; et le grand Schalitz est un Allemand en France, pendant l'Occupation. Les personnages féminins partagent également cette identité marginale: Mémère Desjarlais est amérindienne ("Mémère Desjarlais », dans Primeau, 1996), Sophia est handicapée («Sophia », dans Israël, 1997), Tante Moll est une courtisane (sinon une prostituée de luxe) («Tante Moll», dans Israël, 1997), Camilla vit en réfugiée en Irlande («Camilla », dans Israël, 1997), Pépita est une Espagnole vivant au Danemark ("Pépita», dans Israël, 1997). Ce nombre important de personnages marginaux pourrait de nouveau s'expliquer par le parcours des auteurs des recueils, qui ont certainement développé, de par leur statut de déplacé et d'immigrant, une sensibilité particulière à la marginalité. Il pourrait également être le résultat de l'esthétique du genre, alors que le personnage problématique, en marge de la société, s'avère "intéressant » à faire évoluer dans une histoire courte où les effets de surprise sont recherchés. Ajoutons également que des personnages stéréotypés (le personnage juif pendant l'Occupation, l'Espagnole vivant dans un pays scandinave, l'Amérindienne vivant au sein d'une communauté blanche, l'immigrant, le handicapé, etc.) favorisent une cristallisation plus rapide de l'intrigue autour d'une sensibilité culturelle. Si les gens heureux n'ont pas d'histoire, un personnage issu d'un milieu marginal, dans nos sociétés francophones minoritaires, se prête mieux à une identification de la part des lecteurs. N'oublions finalement pas l'économie du récit ainsi réalisée. En utilisant un stéréotype de façon à mieux exprimer la marginalité du personnage, le narrateur n'a pas à tout expliquer à son lecteur. Il est clair que les francophones du Canada peuvent s'imaginer facilement les difficultés rencontrées par les Amérindiens qui décidèrent de 
vivre parmi les blancs, au XXe siècle, tout en conservant leur culture. L'utilisation de l'image stéréotypée à portée négative est ainsi inversée, et sert alors de déclencheur à un récit qui engage une réflexion par rapport à l'exclusion ou à la persécution, ou encore qui offre un nouvel espoir à l'exclus.

\section{Les nouvelles racontant le monde de l'autre}

Le monde de l'autre, dans le cas des auteurs à l'étude, est en fait celui de leur terre d'accueil en Colombie-Britannique. MarieFrance Auger (2005), dans le premier mémoire de maîtrise écrit sur la littérature d'expression française en ColombieBritannique, a développé une hypothèse selon laquelle la nature était d'une grande importance pour les personnages francophones vivant dans la province, et permettait une stabilisation des quêtes identitaires. Il semble effectivement que le fait de situer l'action des personnages des nouvelles sur la côte du Pacifique ait eu une influence déterminante sur les thèmes abordés et le décor des nouvelles de mon corpus. Pourrait également s'ajouter à cette hypothèse une perspective théorique liée à l'appréhension de l'autre développée par Simon Harel dans son ouvrage sur les braconnages identitaires. Les braconnages organisés par les auteurs que j'étudie pourraient être associés à ce que le chercheur montréalais tente d'isoler :

J'ai évoqué la figure du braconnage afin de faire entendre la singularité d'une posture qui recourt à la ruse et à la dissimulation pour mieux piéger le bien d'autrui dans un monde aux contours incertains. Peu à peu, la figure du braconnier est devenue l'incarnation d'une chasse heureuse au cœur du monde des signes. (p. 123-124) 
Il y aurait donc un passage obligé, dans les modalités d'écriture, permettant aux nouvellistes d'intégrer une société d'accueil parfois bien différente de leur expérience de vie.

L'une des premières différences qui doit être notée est l'absence de passé : absence de souvenirs des auteurs dont la mémoire retient plutôt les expériences de vie de la société de départ, et absence de mémoire de la province de la ColombieBritannique, qui est une province très jeune dont la communauté francophone n'a pas encore complètement reconstitué son histoire. L'utilisation de personnages stéréotypés qui permettront une réflexion sur l'altérité peut cependant toujours être remarquée. La nouvelle de MargueriteA. Primeau «Ol' Man, Ol' Dog et l'enfant» met en scène les réflexions d'un vieil homme, ancien professeur d'université, son chien, mais également un jeune enfant qui bégaie et qui souffre vraisemblablement d'un handicap mental. L'enfant devient, au cours de la nouvelle, le Fils de Cybèle, celui qui règne de façon symbolique sur la forêt. En revanche, des nouvelles vont utiliser le stéréotype de façon à accélérer la vitesse de la narration et à mieux préparer le retournement final du récit bref. Je donnerais comme exemple de nouvelles de cette catégorie "Manuel le macho » (Castex, 2005), où un bel Andalou de Vancouver perd sa virilité, et « La femme en or » (ibid.), où une jeune obèse suit un régime d'amaigrissement drastique avant de gagner, dans un concours, son poids en or.

On trouve également des nouvelles où la surprise $^{2}$ devient l'élément central, alors que toute la narration est justement axée sur un retournement de situation. Les nouvelles de Jean-Claude Castex sont fort bien ciselées à cet

2 Voir, à propos de cette question, Patrick Imbert (2000). 
égard. Pour ne donner qu'un exemple, pensons à la nouvelle "Le fantôme », où un homme est persécuté par ce qu'il croit être le fantôme d'une jeune fille. On découvre cependant, à la fin de la nouvelle, que le "fantôme » était en fait la sœur d'une victime d'acte criminel qui s'était méprise sur l'identité du personnage principal, et voulait ainsi venger sa sœur décédée dans des conditions atroces. Ces nouvelles s'inspirent souvent de genres parallèles, comme le fantastique et le polar.

L'inquiétude apparaît également dans les nouvelles britanno-colombiennes. Les situations alors évoquées démontrent l'impuissance des personnages face au destin et à une société dont on ne comprend plus très bien les règles. Pensons par exemple à la nouvelle de Claude Bouygues intitulée «Vancouver, la ville du -ing»; la métropole de la ColombieBritannique devient alors un arrêt incontournable (et final) pour les suicidés du monde entier, et des entreprises vont même jusqu'à aider les pauvres hères à commettre l'impensable. Même ton, mais cette fois-ci lié à la sexualité, dans une autre nouvelle du même auteur, "La décadence, catalogue ». Le lecteur est ici convié à suivre Lana-Lise dans ses ébats sexuels les plus rocambolesques. Vancouver se transforme alors de façon métaphorique, pour l'un des personnages, en «immense flaque de l'Océan». «La contravention » et "L'évaluation», deux nouvelles de JeanClaude Castex, participent d'un même mouvement lié aux aléas de la vie et en partie au fantastique. Dans "La contravention", un policier est secoué par la prédiction (qui s'avérera juste) de sa mort effectuée par une automobiliste, alors que «L'évaluation» raconte les affres d'une enseignante dont les étudiants, brillants, décident, lors de la visite de l'inspecteur général, de ne dire mot. Ces nouvelles rappellent une autre 
caractéristique des braconnages identitaires qui implique un jeu des narrateurs ou des personnages avec des situations dangereuses dans un lieu encore peu connu :

Braconner, c'est se situer en un lieu où l'on s'expose à être piégé, à devenir, par un subit retournement de situation, celui qui est chassé et qui, pour cette raison, ruse avec la loi à ses risques et périls. (Harel, 2006, p. 63)

Bien évidemment, ce piège où se laissent enfermer les personnages implique une réaction. Dans les cas qui m'intéressent, elle ne permet pourtant pas aux protagonistes de vaincre les forces du lieu que l'on braconne ou même de s'en émanciper. Il faut dans cette perspective considérer la puissance que représente le destin tragique auquel certains personnages sont condamnés. Simon Harel va d'ailleurs souligner l'importance de cataclysmes émotionnels qui «donnent forme à nos trajectoires ${ }^{3}$ ». Il voit également ces violences symboliques, psychiques et physiques, comme des éléments positifs à l'apparition de l'identité 4 . On assiste alors à une double lecture des événements. Dans un premier temps, l'on ne parvient que difficilement à comprendre les règles de la nouvelle société, le destin semblant se retourner contre les personnages. Dans un second temps, les nouveaux arrivants soumettent à de véritables « tests » la société d'accueil.

\footnotetext{
3 « Si le territoire est un lieu d'accueil, s'il est un lieu d'expression, s'il permet la création de microévénements qui donnent forme aux braconnages, il est de plus opportun de revendiquer l'existence de petits cataclysmes émotionnels qui donnent forment à nos trajectoires » (Harel, 2006, p. 123).

4 «Pour ces raisons, la référence aux braconnages identitaires suppose que l'on interroge les violences symboliques, psychiques et physiques qui sont autant de refus de l'universalisme abstrait de la raison pluriculturelle » (Harel, 2006, p. 116).
} 
Il faudrait aussi se pencher sur la dimension inconsciente $\mathrm{du}$ processus de braconnage et aux pulsions internes qui s'emparent du Génie du lieu. Une demi-douzaine de nouvelles pourrait correspondre à ce parcours. La dimension émotive s'inscrit alors au sein même des objets ou des animaux : un chat que l'on abandonne dans la forêt et qui est finalement tué sur la route devant ses propriétaires qui, rongés de remords, essayaient de le retrouver («Jonas », dans Castex, 2007); l'eau d'un village non loin de Kamloops (dans la vallée de l'Okanagan), fort bonne au goût, qui s'avère provenir du cimetière («L'eau c'est la vie», dans Castex, 2007); le nougat qui colle symboliquement un mari infidèle à son épouse (« Le nougat», dans Israël, 1997); et finalement «Les ombres » et « Le totem », deux nouvelles respectivement d'Inge Israël et de Marguerite-A. Primeau, qui relèvent de l'obsession fraternelle. Dans «Les ombres », un jeune garçon, Sammy, développe une peur incontrôlée de son frère, Richard. L'ombre même de ce dernier, devenu jeune délinquant, terrorisera Sammy. C'est finalement un accident, alors que Richard sera gravement blessé, près de la Siwash Rock, dans le parc Stanley, à Vancouver, qui mettra fin à cette terreur maladive. Dans «Le totem ", c'est un objet hérité de Mariette, la demi-sœur du personnage principal, qui pousse Jeanne dans les affres de l'inquiétude. Alors que Mariette, jusqu'à sa mort, est persuadée que le totem lui apporte une protection spéciale, Jeanne, acculée à la pauvreté, meurt après s'être finalement convaincue qu'elle devait vendre l'objet mystérieux. Ces nouvelles expriment de façon assez claire comment des pulsions (remords, peurs incontrôlées, mort, objet possédant des pouvoirs magiques, pulsions érotiques) s'expriment grâce à des objets ou à des symboles. Rien de surprenant, alors, de 
constater que ces objets se retournent contre leurs personnages, le totem emportant Jeanne dans la mort, le nougat qui colle aux dents rappelant l'homme infidèle à la réalité, Jonas se vengeant de ses maîtres en les rendant témoins de sa fin atroce, l'eau si bonne à boire se révélant transporter le goût de la mort. Ces retournements impliquent bien entendu une perte immédiate pour les personnages principaux de ces nouvelles. S'ils sont parfois débarrassés de l'élément gênant, ils sont le plus souvent précipités dans la mort, ou encore bien en place pour la surprise de leur vie.

\section{Conclusion}

J'ai tenté de démontrer dans cet article que le braconnage identitaire, et son corollaire, la littérature du soi, se sont jusqu'à aujourd'hui côtoyés dans la littérature francophone de la Colombie-Britannique. Cette double perspective narrative explique l'existence de recueils "bicéphales», c'est-à-dire de recueils de nouvelles comportant à la fois des récits à propos du soi et du passé, et des narrations s'immisçant dans le présent et le lieu de l'autre. Un échantillon de ces recueils publiés entre 1988 et 2007 me permet de croire que la composition de ces ouvrages laisse une place de plus en plus grande au braconnage identitaire (du moins en nombre de nouvelles). Ce phénomène peut s'expliquer, bien entendu, par la biographie des auteurs qui ont vécu leur enfance et leurs premières années à l'étranger ou dans une autre partie du Canada, avant de venir s'établir en ColombieBritannique. On pourrait aussi évoquer, afin d'expliquer ce phénomène, une lente prise de conscience, à partir des années 1980 et 1990, d'une francophonie bien distincte en ColombieBritannique. 
Il faut cependant noter que les nouvelles du soi, celles du passé, reflètent une dimension autobiographique et une réflexion humaniste à propos de la pauvreté, de la guerre, du destin des exclus, etc. On s'y sent en pleine possession des repères identitaires, même si la pauvreté, la misère et l'injustice sont mises en scène. Il existe, pour ces personnages, un ordre humain qui, quoique temporairement bouleversé par la guerre, l'occupation ou l'éloignement, sera rétabli. En revanche, les nouvelles se déroulant en Colombie-Britannique, alors que l'identité se braconne, sont, d'un point de vue humain, beaucoup plus inquiétantes. Les objets, le destin, l'ironie du sort, les renversements narratifs, les pulsions sexuelles, toutes ces dimensions surgissent, abandonnant souvent les protagonistes et les lecteurs dans une situation terminale dramatique, sinon tragique. Cette chute narrative, aboutissant à une vision d'horreur (personnage volé ou ruiné) ou à une constatation morbide (personnage décédé, atteint du Sida), ne permet au processus identitaire d'avancer qu'au compte-gouttes dans un monde aux modalités inquiétantes. S'il faut en déduire quelque chose, c'est probablement que le processus de braconnage n'en est encore qu'à ses débuts, même si le pourcentage de nouvelles dont l'action se déroule en ColombieBritannique augmente d'un recueil à l'autre. La facture des nouvelles de l'autre ne comporte cependant que peu de similitudes avec celles des nouvelles du soi. Quelques exceptions, comme « Ol' Man, Ol' Dog et l'enfant » (Primeau, 1996), pourraient laisser croire que les thématiques des nouvelles du soi ont commencé à migrer vers les nouvelles de l'autre. Mais ce mouvement, du moins pour l'instant, est ténu. Il faudra attendre encore quelques années avant que le braconnage ne se modifie et affranc(h)ise (sic) la côte du Pacifique. 


\section{Bibliographie}

AUGER, Marie-France (2005), «Une étude de la littérature francophone de la Colombie-Britannique», mémoire de maîtrise, Burnaby, Simon Fraser University.

BouyGuEs, Claude (1999), De parts et d'autres, Paris, L'Harmattan, coll. «Écritures».

BOYER, Jean-Claude (2000, 2002-2005), Nouvelles d'ici et d'ailleurs, 5 tomes, Montréal, Éditions Francine Breton.

CASTEX, J.-C. (1988), Le Gros lot, Saint-Boniface, Éditions des plaines.

- (2005), Le Fantôme et autres histoires vraies, White Rock, Phare Ouest.

— (2007), C'est arrivé un jour!, Ottawa, L'Interligne, coll. « Vertiges ».

HAREL, Simon (2006), Braconnages identitaires. Un Québec palimpseste, Montréal, VLB éditeur, coll. « Le Soi et l'Autre ».

HAUTBERG, M.-F. (2001), Anthologie de nouvelles francophones de la Côte-Pacifique du Canada, White Rock, Phare-Ouest.

IMBERT, Patrick (2000), "La surprise du texte bref », dans Guy Poirier et Pierre-Louis Vaillancourt (dir.), Le Bref et l'instantané: à la rencontre de la littérature québécoise du XXIe siècle, Orléans, David, p. 75-95

ISRAËL, Inge (1997), Le Tableau rouge, Ottawa, Vermillon, coll. « Parole vivante».

KELETT-BETSOS, Kathleen (2007), «La nouvelle francophone en Colombie-Britannique: un genre mineur en milieu minoritaire», dans Guy Poirier (dir.), Culture et littérature francophones de la Colombie-Britannique: du rêve à la réalité, Ottawa, David, coll. « Voix savantes », p. 201-227.

OUELLET, Pierre (2007), "Le principe d'altérité », dans Pierre Ouellet et Simon Harel (dir.), Quel Autre? L'altérité en question, Montréal, VLB éditeur, coll. « Le soi et l'autre», p. 7-43. 
PoIRIER, Guy (2009), «Habiter et rêver la ColombieBritannique francophone», dans Lucie Hotte et Guy Poirier (dir.), Habiter la distance. Études en marge de La distance habitée, Sudbury, Prise de parole, p. 81-100.

PRIMEAU, Marguerite-A. (1988), Le Totem, Saint-Boniface, Éditions des Plaines.

- (1996), Ol'Man, Ol'Dog et l'enfant ou autres nouvelles, SaintBoniface, Éditions du Blé.

RECIZAC, C. (1995), Nouvelles d'ici... et d'ailleurs, Surrey, Phare Ouest.

\section{Résumé}

Cet article étudie sept recueils de nouvelles écrits par des auteurs francophones de la Colombie-Britannique entre 1988 et 2007. Grâce à la théorie du braconnage identitaire développée par Simon Harel, il permet de mieux saisir les caractéristiques de ces recueils où sont regroupées des nouvelles reflétant le monde du soi des auteurs (leur passé, leurs lieux d'origine) et les nouvelles établissant un dialogue avec l'autre, c'est-à-dire avec le territoire de la Colombie-Britannique.

\section{Abstract}

This paper analyzes seven collections of short stories written by British Columbian Francophone authors from 1988 to 2007. Taking its cue from Simon Harel's notion of "poaching" as applied to identity constructions, it provides a deeper understanding of the characteristics of these collections which bring together stories that explore the authors' world of the self (their past, their origins) and stories that engage a dialogue with the other, that is, with the territory of British Columbia. 